\title{
Regeneración natural de nueve especies maderables en un bosque intervenido de la Amazonia Boliviana
}

\begin{abstract}
Jhon Wilber Leigue GÓMEZ
RESUMEN

Un aspecto fundamental del manejo forestal sostenible, es el mantenimiento de la regeneración natural en los bosques aprovechados. A corto y largo plazo, el aprovechamiento forestal tiene varias consecuencias, sobre la regeneración natural de las especies forestales. Esta investigación, compara la abundancia de la regeneración natural y la distribución espacial de plántulas de nueve especies maderables, entre un bosque aprovechado y un testigo. La abundancia y estructura espacial, fue determinada mediante 55 parcelas de muestreo anidadas $(20 \times 20 \mathrm{~m}, 10 \times 10 \mathrm{~m})$, estas fueron distribuidas en 180 hectáreas en cada condición de bosque evaluado. Dentro de las parcelas de $10 \times 10 \mathrm{~m}$, se registraron las categorías: plántula, brinzal y latizal. En las parcelas de 20 x 20, se midieron los árboles semilleros con DAP $\geq 20 \mathrm{~cm}$. En bosque aprovechado C. racemosa, T. altissima y $A$. lecointei mostraron mayor abundancia total. Según categorías de regeneración, la abundancia de P. corymbosum resultó mayor en bosque aprovechado; pero en las especies $C$. racemosa, T. altissima, D. odorata y $C$. micrantha fue mayor en el bosque testigo. La abundancia de $A$. lecointei, P. heterophylla y Virola sp. no difirió entre sitios. Espacialmente, solo A. lecointei y Virola sp mostraron patrones diferentes entre condiciones de bosque estudiados. Estos resultados, permiten concluir que el aprovechamiento forestal, no modifica significativamente la abundancia y estructura espacial de la regeneración de todas las especies forestales, por lo que, las intensidades de extracción moderada no comprometería el potencial de la regeneración natural en bosques manejados.
\end{abstract}

PALABRAS-CLAVE: Regeneración natural, abundancia, bosque aprovechado, especies forestales, patrón espacial.

\section{Natural regeneration of nine commercial timber species in a selectively logged forest in the Bolivian Amazon}

\begin{abstract}
A key attribute of sustainable forest management is the natural regeneration of exploited tree species in selectively logged forests. Selective logging has various consequences for natural regeneration, both on the short and the long term. In this study, the recruit abundance and spatial distribution of nine exploited tree species was determined, and compared between a logged and an undisturbed forest area in the Bolivian Amazon. The recruit abundance and spatial distribution was evaluated in 55 nested plots $(20 \times 20 \mathrm{~m}, 10 \times 10 \mathrm{~m})$ within 180 ha of forest, in each of the two study sites. Within the $10 \times 10 \mathrm{~m}$ plots, seedlings, saplings and pole-sized trees were searched, measured and located. In the 20 x $20 \mathrm{~m}$ plots, reproductive individuals of $>20 \mathrm{~cm}$ Diameter at Breast Height (DBH) were included. In the exploited forest, total abundance of C. racemosa, T. altissima and $A$. lecointei was higher than in the undisturbed forests. In some of the categories recruits of $P$. corymbosum were more abundant in the exploited forest, while the reverse was found for certain size categories of C. racemosa, T. altissima, D. odorata and $C$. odorata. The recruit abundance of A. lecointei, P. heterophylla and Virola sp. did not differ between forest sites. As for the spatial distribution, only $A$. lecointei and Virola sp. showed differences patterns between forest sites. These results suggest that selective logging, as applied at the study site, did not strongly change abundance and spatial distribution of exploited tree species. Probably, low intensity of selective logging does not hamper natural regeneration of exploited tree species.
\end{abstract}

KEYWORDS: Bolivia, natural regeneration, selective logging, spatial distribution, tropical forest.

1'Asociación PROMAB,Riberalta, Beni, Bolivia, Final Avenida Ejército Nacional, Campus Universitário (UAB), E-mail: asoc_promab@yahoo.com 


\section{INTRODUCCIÓN}

Una de las bases fundamentales del manejo sostenible de los bosques tropicales, es el mantenimiento de la regeneración natural. Esta forma de manejo, requiere que las especies maderables aprovechadas, regeneren de forma natural para mantener sus poblaciones y asegurar la futura productividad del bosque (Bawa \& Seidler, 1998; Mostacedo \& Fredericksen, 1999).

El aprovechamiento de especies maderables, tiene varias consecuencias para la regeneración natural, algunas con efectos opuestos. Por un lado, los dańos y mortalidad de los individuos, la reducción considerable de la producción de frutos, y el aumento de la disponibilidad de luz en el bosque residual (Chapman \& Chapman ,1997; Curran et al., 1999; Dickinson et al., 2000; Jackson et al., 2002; Van Rheenen et al., 2004). Por otro lado, se ha encontrado que el aprovechamiento forestal y otras actividades antropogénicas, podrían influenciar la distribución espacial de las especies del bosque (Clark et al. 1995; Rivas et al, 2005); incluso puede alterar significativamente la abundancia y modificar la distribución espacial de la regeneración natural de las especies aprovechadas y remanentes. Sin embargo, a nivel de especies, aun no se conoce a ciencia cierta, cuál es el grado de impacto que ocasiona la remoción de una alta proporción de árboles semilleros y cómo se configura la estructura espacial, ante un escenario pos aprovechamiento con menos árboles semilleros y más distanciados entre sí, cuyos temperamentos y estrategias reproductivas son diversos. Este tipo de información es necesaria, para mejorar las prescripciones genéricas de manejo forestal existentes.

El impacto integrado de estos efectos, se puede evaluar solamente algunos años después del aprovechamiento forestal. Frecuentemente, los estudios de los efectos del aprovechamiento sobre la regeneración natural de algunas especies, han sido realizados inmediatamente después del aprovechamiento (Fredericksen \& Mostacedo, 1999; Pariona et al., 2003; Schiotz et al., 2006). Pero dichos estudios, no contemplan el impacto del aprovechamiento forestal a largo plazo. Además, para una gran mayoría de las especies forestales comerciales, la información es aun escasa y en otros casos inexistente (Mostacedo \& Fredericksen, 1999).

Si bien los estudios que midan los efectos del manejo forestal, son difíciles de realizar experimentalmente con suficientes replicas; también es cierto, que se puede aprender bastante basado en observaciones y comparaciones entre condiciones de bosques. En este estudio, se comparan la abundancia de la regeneración natural y la distribución espacial de plántulas de nueve especies maderables entre un bosque aprovechado y un bosque natural.

\section{MATERIALES Y MÉTODOS}

\section{Área de estudio}

El sitio de estudio "El Verdum", es un bosque bajo manejo perteneciente a la comunidad 12 de Octubre, está ubicado en el norte amazónico de Bolivia (1055’05”Sur, 65040'50"Oeste). Según la clasificación bioclimática de Holdridge et al. (1971), se trata de un bosque húmedo tropical, con una altura promedio de $32 \mathrm{~m}$ y arboles emergentes que llegan a 50 metros (Zuidema, 2003). La precipitación media anual es de $1700 \mathrm{~mm}$ y $27^{\circ} \mathrm{C}$ de temperatura media, con una marcada diferencia entre la época lluviosa (diciembre a abril) y seca (mayo a noviembre).

El Verdum abarca una superficie total de 2511 hectáreas. Según el inventario forestal de reconocimiento, se registraron 116 especies, de las cuales nueve fueron palmeras y 107 árboles con diámetro a la altura del pecho (DAP) $\geq 20 \mathrm{~cm}$. Se encontró una abundancia de 160 árboles (DAP $\geq 20 \mathrm{~cm}$ ), área basal de $24 \mathrm{~m}^{2}$ y el volumen de madera en tronca fue de $239 \mathrm{~m}^{3}$.ha ${ }^{-1}$. El Plan de Manejo de la comunidad, contempla ciclos de corta de 25 años, en principio se destinó una superficie de 500 ha como primer área de aprovechamiento. Posteriormente se realizarán diez intervenciones periódicas en áreas de 180 ha. (Zonta, 1999). En el año 2000, se realizó el primer aprovechamiento forestal sobre una superficie de 500 hectáreas. En esa oportunidad, se aprovecharon 21 especies arbóreas y se extrajo un volumen total de $8082 \mathrm{~m}^{3}$ de madera en rola. Nueve de estas especies fueron objeto del presente estudio (Tabla 1).

\section{Diseño de muestreo}

Dentro del área de manejo, se seleccionaron dos áreas anuales de aprovechamiento forestal (AAA), procurando homogeneizar el tamaño de las áreas seleccionadas a 180 hectáreas. Así, en el año 2007 se instaló una dentro de las 500 ha intervenidas; $y$ la otra en una área contigua, medida antes del aprovechamiento forestal. En cada sitio, se establecieron 11 brechas equidistantes y por cada brecha se dispusieron aleatoriamente 5 parcelas cuadradas sobrepuestas, quedando así las muestras distribuidas por toda el área. Las parcelas utilizadas fueron de $20 \times 20 \mathrm{~m}$ y dentro de ésta una subparcela de $10 \times 10 \mathrm{~m}$.

\section{Toma de datos}

En las sub-parcelas de $10 \times 10 \mathrm{~m}$, se registraron todos los individuos con altura $\leq 0,30 \mathrm{~m}$ (plántula); individuos $>0.3$ $\mathrm{m}$ hasta $1.3 \mathrm{~m}$ altura y DAP $\leq 5 \mathrm{~cm}$ (brinzal); e individuos > $1.3 \mathrm{~m}$ de altura y $5 \mathrm{~cm}>$ DAP $\leq 10 \mathrm{~cm}$ (latizal), además del nombre común de las nueve especies estudiadas. Mientras que en la parcelas de $20 \times 20 \mathrm{~m}$, se registraron todos los árboles semilleros con DAP $\geq 20 \mathrm{~cm}$. En el presente estudio no se registró la categoría de fustal $10 \mathrm{~cm}>\mathrm{DAP}<20 \mathrm{~cm}$. 
Tabla 1 - Características ecológicas, abundancia y densidad de apeo de las nueve especies forestales evaluadas en el área de manejo El Verdum (bosque intervenido y no intervenido). La abundancia corresponde a los árboles semilleros, con diámetro mayor 0 igual a $40 \mathrm{~cm}$, y los árboles aprovechados, con diámetro mayor o igual al diámetro mínimo de corta (DMC: según la normativa boliviana mayor o igual a $50 \mathrm{~cm}$ ).

\begin{tabular}{|c|c|c|c|c|c|c|}
\hline \multirow{2}{*}{ Especies } & \multirow{2}{*}{ Familias } & \multirow{2}{*}{ Gremios Ecológicos } & \multirow{2}{*}{ Dispersión } & \multicolumn{2}{|c|}{ Abundancia $\left(\right.$ ha $\left.^{-1}\right)$} & \multirow{2}{*}{$\begin{array}{l}\text { Arboles }\left(\text { ha }^{-1}\right) \\
\text { aprovechados }\end{array}$} \\
\hline & & & & Intervenido & No intervenido & \\
\hline Astronium lecointei & Anacardiaceae & Heliófita durable & Viento & 9.09 & 9.09 & 0.35 \\
\hline Cariniana micrantha & Lecythidaceae & Heliófita parcial & Viento & 2.27 & 0.00 & 0.21 \\
\hline Clarisia racemosa & Moraceae & Esciofita parcial & Animal & 4.55 & 18.18 & 0.27 \\
\hline Dipteryx odorata & Fabaceae & Esciófita parcial & Animal & 2.27 & 4.55 & 0.14 \\
\hline Hymenaea courbaril & Caesalpineaceae & Esciófita parcial & Animal & 0.00 & 4.55 & 0.07 \\
\hline Peltogyne heterophylla & Caesalpnioideae & Esciófita parcial & Viento & 0.00 & 6.82 & 0.13 \\
\hline Pithecellobium corymbosum & Mimosaceae & Heliófita durable & Viento & 0.00 & 13.64 & 0.25 \\
\hline Tetragastris altissima & Burseraceae & Esciófita & Animal & 18.18 & 40.91 & 0.20 \\
\hline Virola sp. & Myristicaceae & Esciófita & Animal & 4.55 & 2.27 & 0.21 \\
\hline
\end{tabular}

\section{Análisis estadístico}

Para contrastar la abundancia de las categorías de regeneración natural (plántula, brinzal y latizal), entre condiciones de bosque (intervenido y no intervenido), se utilizó la prueba Chi-cuadrado (Tabla de contingencia). La abundancia total (plántula, brinzal y latizal juntas) entre especies, se contrastó mediante el análisis de varianza (ANOVA). Mientras que para probar o rechazar la aleatoriedad de la distribución espacial de las plántulas (ocurrencia en las 55 parcelas de muestreo), se comparó con la distribución de Poisson (Upton \& Fingleton, 1985), mediante la prueba de Kolmogorov-Smirnov.

\section{RESULTADOS}

\section{Abundancia de la regeneración natural}

Para el bosque aprovechado, las tres especies más abundantes fueron: C. racemosa, T. altissima y A. lecointei, mientras que las menos abundantes fueron: $H$. courbaril, $D$. odorata. y C. micrantha (Figura 1). Es decir, que la variación inter específica de la abundancia total (las tres categorías juntas), fue significativa (ANOVA, $F=5,401, p<0,01$ ). Mientras que la abundancia entre categorías de tamaño y entre grupos de especies esciófitas y heliófitas no presentó diferencias estadísticas. Sin embargo, la abundancia de algunas categorías de regeneración natural en cinco especies, difirió significativamente entre el bosque intervenido y no intervenido. Sólo en $P$. corymbosum, las categorías de plántula y brinzal $\left(\chi^{2}=18,56 \mathrm{P}<0,05\right)$ mostraron mayor abundancia en bosque intervenido. Mientras que $C$. racemosa en las categorías de plántula, brinzal y latizal $\left(\chi^{2}=6,27 \mathrm{P}<0,05\right)$; T. altissima en brinzal y latizal $\left(\chi^{2}=826 \mathrm{P}<0,05\right) ; D$. odorata en plántula, brinzal y latizal $\left(\chi^{2}=12,03 \mathrm{P}<0,05\right)$, y $C$. micrantha en plántula y brinzal $\left(\chi^{2}=8,21 \mathrm{P}<0,05\right)$ mostraron mayor abundancia en bosque no intervenido. Por otro lado, la abundancia de las especies $A$. lecointei, $P$. heterophylla, $H$. courbaril y Virola sp., no difirió entre condiciones de bosques estudiados.

\section{Distribución espacial de las plántulas entre condiciones de bosque}

Basado en la Figura 2, la distribución de frecuencias de las plántulas en las parcelas de muestreo, reveló que las plántulas se distribuyeron espacialmente en agregados y aleatoriamente. Asíla distribución espacial de las plántulas de dos especies forestales, alternó entre patrón espacial aleatorio y agregado, dependiendo de la condición de bosque estudiado. A. lecointei con patrones aleatorio/agregado y Virola sp. con patrones agregado y aleatorio respectivamente. En tanto que las restantes siete especies, mostraron un determinado patrón espacial en ambas condiciones de bosques. Así las especies C. micrantha, P. corymbosum, $P$. heterophylla e $H$. courbaril mostraron una distribución aleatoria. Pero las especies $T$. altissima, C. racemosa y D. odorata con un mismo patrón agregado (Tabla 2).

Tabla 2 - Resumen estadístico de los patrones espaciales de las especies forestales en bosque intervenido y no intervenido.

\begin{tabular}{lccc}
\hline Especies & $\begin{array}{c}\text { Bosque } \\
\text { intervenido }\end{array}$ & $\begin{array}{c}\text { Bosque no } \\
\text { intervenido }\end{array}$ & Patrón espacial \\
\hline A. lecointei & $Z=1,05 p>0,05$ & $Z=1,46 p<0,05$ & $\begin{array}{c}\text { Aleatorio, } \\
\text { agregado }\end{array}$ \\
Virola sp. & $Z=1,58 p<0,05 \quad Z=0,04 p>0,05$ & $\begin{array}{c}\text { Agregado, } \\
\text { aleatorio }\end{array}$ \\
C. micrantha & $Z=0,11 p>0,05$ & $Z=0,16 p>0,05$ & Aleatorio \\
P. corymbosum & $Z=0,62 p>0,05$ & $Z=0,01 p>0,05$ & Aleatorio \\
P. heterophylla & $Z=0,94 p>0,05$ & $Z=1,07 p>0,05$ & Aleatorio \\
H. courbaril & $Z=0,001 p>0,05$ & $Z=0,16 p>0,05$ & Aleatorio \\
T. altissima & $Z=3,55 p<0,05$ & $Z=1,97 p<0,05$ & Agregado \\
C. racemosa & $Z=2,31 p<0,05$ & $Z=2,11 p<0,05$ & Agregado \\
D. odorata & $Z=1.42<0,05$ & $Z=1,44 p<0,05$ & Agregado \\
\hline
\end{tabular}



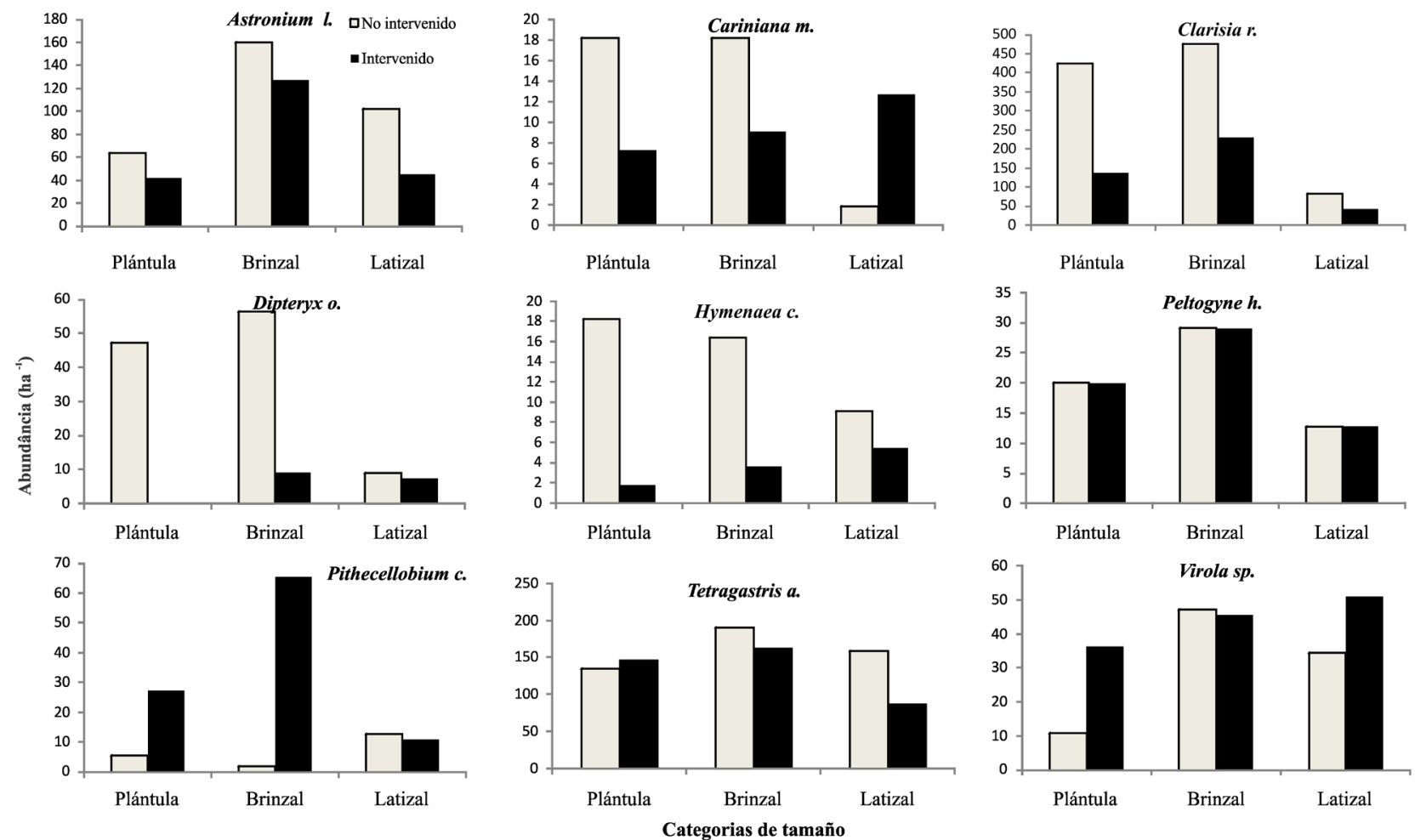

Figura 1 - Abundancia de las categorías de regeneración natural: plántula (altura $\leq 0,30 \mathrm{~m})$, brinzal $(>0,30 \mathrm{~m}$ altura $\leq 1,3 \mathrm{~m}$; DAP $\leq 5 \mathrm{~cm})$ y latizal (altura $>1,3 \mathrm{~m} ; 5 \mathrm{~cm}>\mathrm{DAP} \leq 10 \mathrm{~cm}$ ) de nueve especies forestales, entre el bosque intervenido y no intervenido en "El Verdum", Beni, Bolivia.

\section{DISCUSIÓN}

\section{Regeneración natural en bosques aprovechados}

Experiencias de manejo forestal en bosques tropicales revelan que el aprovechamiento forestal afecta de manera distinta la abundancia de las especies comerciales (Magnusson et al., 1999; Pimentel et al., 2002). Incluso a ocho años de la intervención de El Verdum, la abundancia de la regeneración natural se mostró ostensiblemente variable entre las especies aprovechadas (Figura 1); confirmando que las especies forestales están fuertemente influenciada por la disponibilidad de luz, formación de claros y competencia con las especies pioneras del bosque (Denslow, 1987; Canham, 1989; Pariona et al., 2003).

El incremento de la regeneración natural como respuesta a las intervenciones, ha sido observado en varias especies clasificadas como pioneras de larga vida (Graaf et al., 1999; Rose, 2000; Arets et al., 2003; Peña-Claros et al., 2008). Paradójicamente, las especies más abundantes fueron las esciófitas $(C$. racemosa y $T$. altissima, ambas con dispersión animal y solo una heliófita (A. lecointei), con dispersión eólica (Tabla 1).

Debido a que los árboles semilleros residuales de estas especies juntas alcanzaran el $78 \%$ del total, y un promedio de 0,3 árboles aprovechados ha ${ }^{-1}$; en contraste con la menor regeneración de $H$. courbaril, $D$. odorata y $C$. micrantha (11 $\%$ de semilleros y 0,1 árboles aprovechados $\mathrm{ha}^{-1}$ ) sugiere la existencia de una cierta dependencia entre la abundancia de la regeneración natural y los árboles semilleros residuales; excepto con el temperamento, tipo de dispersión y la tasa de remoción en este grupo de especies.

Pero otras esciófitas como P. heterophylla y Virola sp., con dispersión animal y eólica, exhibieron una abundancia intermedia; en apariencia indiferente a la intervención. Similar comportamiento ha sido observado en otras especies del mismo temperamento (Pimentel et al., 2002; Peña-Claros et al., 2008).

En contraste, la abundancia de la regeneración natural de cinco de las nueve especies forestales, difirió estadísticamente entre condiciones de bosques. A pesar que $P$. corymbosum resultó mayor en bosque intervenido, y $C$. racemosa, $T$. altissima, D. odorata, y C. micrantha en bosque no intervenido. No se puede descartar la influencia en los resultados de las diferencias naturales entre sitios evaluados, que pudieran enmascarar los verdaderos efectos del aprovechamiento. En cualquier caso, este probable sesgo exige la interpretación cautelosa de los hallazgos, debido a que en el estudio no se aplicó un diseńo sofisticado (medición antes/después). 

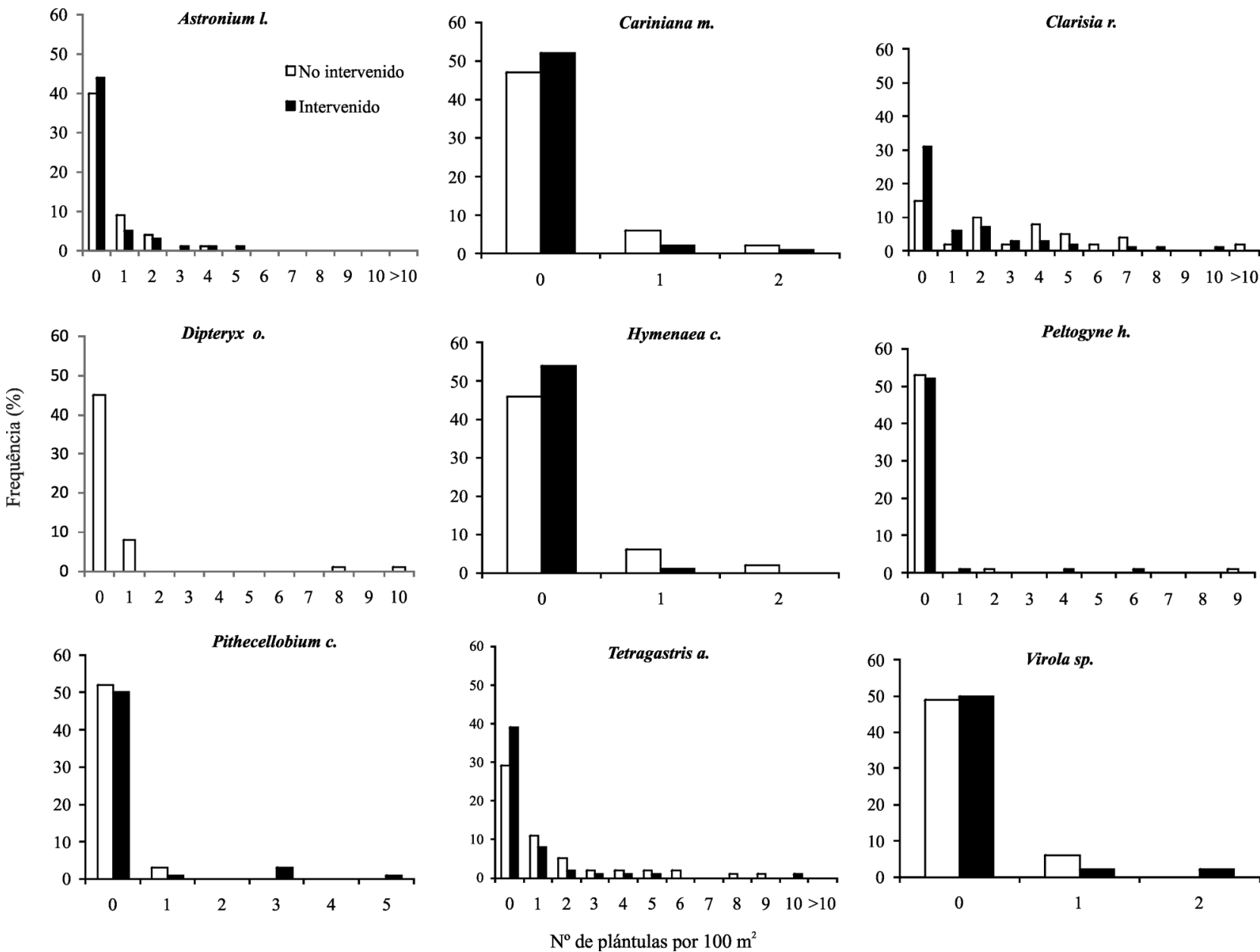

Figura 2 - Frecuencia de plántulas en las parcelas de muestreo $\left(100 \mathrm{~m}^{2}\right)$ en bosque intervenido y no intervenido de El Verdum, Beni, Bolivia.

\section{Patrón espacial de las plántulas en bosques aprovechados}

La heterogeneidad espacial derivada de perturbaciones que eliminan árboles dominantes en el dosel forestal puede condicionar la regeneración natural (Turner y Franz, 1985; He et al., 1997; Dalling et al., 1998). En el bosque aprovechado del presente estudio, las especies que presentaron patrón agregado (T. altissima, C. racemosa, D. odorata. y Virola sp.) y las especies que exhibieron patrón aleatorio (A. lecointei, $C$. micrantha, $P$. corymbosum., $P$. heterophylla, $e H$. courbaril) al parecer muestran cierta correlación con el tipo de dispersión animal y eólica respectivamente (Tablas 1,2 ). Correlación que ha sido observada en una gran mayoría de especies tropicales (Peres \& Baider,1997; Seidler \& Plotkin, 2006). Por lo tanto, a largo plazo se evidencia que, la configuración espacial de la regeneración natural no solo es dependiente de la creación de los claros resultantes del aprovechamiento, sino que también podría estar fuertemente influenciada por la interacción de los componentes bióticos y abióticos del ecosistema boscoso.
Comparativamente, sólo dos especies (A. lecointei y Virola $s p$.), modificaron su estructura espacial entre condición de bosque. Aunque, en función de su ciclo de vida, se espera que éstas modifiquen su estructura espacial (Stewart y Rose, 1990; Lusk y Ogden, 1992; Duncan, 1993; Ward et al., 1996; Gavin y Peart, 1997; Chen y Bradshaw, 1999; Mast y Veblen, 1999). En cualquier caso, se pone de manifiesto lo dinámico de la configuración espacial en el tiempo, por lo que, el aparente cambio del patrón espacial y abundancia de la regeneración natural de las especies forestales, no se puede atribuir exclusivamente al aprovechamiento forestal.

\section{CONCLUSION}

El estudio aporta evidencias de que el aprovechamiento forestal, no modifica de manera significativa la abundancia y la estructura espacial de la regeneración natural de todas las especies forestales aprovechadas. También, demuestra que la regeneración natural en los bosques aprovechados, continúa después del aprovechamiento forestal; y la remoción moderada 
de árboles no comprometería el potencial de la regeneración natural de las especies comerciales en los bosques manejados.

\section{AGRADECIMIENTOS}

A Pieter Zuidema, Alberto Centellas y a Vincent Voos, por el apoyo en la elaboración y revisión del documento. Al equipo del comité forestal: Esmeralda, Erlin, Claudio, Jorge, Manuel, Yonel, Edmundo, Aldo, Raúl y a toda la comunidad. A la Unión Europea, a la Universidad Autónoma del Beni (UAB) y a la Asociación Programa Manejo de Bosques de la Amazonia Boliviana (PROMAB).

\section{BIBLIOGRAFÍA}

Arets, E.J.J.M.; Van der Hout, P.; Zagt, R. 2003. Responses of tree populations and forest composition to selective logging in Guyana. In: Ter Steege, H. (Ed.), Long-term Changes in Tropical Tree Diversity. Studies from the Guiana Shield, Africa. Borneo and Melanesia. Tropenbos Series 22, Tropenbos International, Wageningen, pp. 95-115.

Bawa, K.S.; Seidler, R. 1998. Natural forest management and conservation of biodiversity in tropical forests. Conservation Biology 12: 46-55.

Canham, C. D. 1989. Different responses to gaps among shade tolerant tree species. Ecology, 70: 548-550.

Clark, D.A.; Clark, D.B.; Sandoval, R.; Castro, M.V. 1995. Edaphic and human effects on landscape-scale distributions of tropical rain forest palms. Ecology, 76:2581-2594.

Chapman, C.; Chapman, L.J. 1997. Forest regeneration in logged and unlogged forests of Kibale National Park, Uganda. Biotropica, 29: 396-412.

Chen, J.; Bradshaw, G.A. 1999. Forest structure in space: a case study of an old growth spruce-fir forest in Changbaishan Natural Reserve, PR China. Forest Ecology and Management, 120: 219-233.

Curran, L.M.; Caniago, I.; Paoli, G.D.; Astianti, D.; Kusneti, M.; Leighton, M.; Nirarita, C.E.; Haeruman, H. 1999. Impact of El Niño and logging on canopy tree recruitment in Borneo. Science, 286: 2184-2188.

Dalling J.W.; Hubbell, S.P.; Silvera, k. 1998. Seed dispersal, seedling establishment and gap partitioning among tropical pioneer trees. Journal of Ecology, 86: 674-689.

Denslow, J. S. 1987. Tropical rainforest gaps and tree species diversity. Annual Review Ecology and Systematics, 18: 431-451.

Dickinson, M.B.; Whigham, D.F.; Hermann, S.M. 2000. Tree regeneration in felling and natural treefall disturbances in a semideciduous tropical forest in Mexico. Forest Ecology and Management, 134: 137-151.

Duncan, R.P. 1993. Flood disturbance and the coexistence of species in a lowland podocarp forest, sowth Westland, New Zealand. Journal of Ecology., 81: 403-416.

Fredericksen, T, S; Mostacedo, B. 1999. Regeneration of timber species following selection logging in a Bolivian tropical dry forest. Forest Ecology and Management, 131: 47-55.
Gavin, D.G.; Peart, D. R. 1997. Spatial structure and regeneration of Tetramerista glabra in peat swamp rain forest in Indonesian Borneo. Plant Ecology, 131: 223-231.

Graaf, N.R.; Poels, R.L.H.; van Rompaey, R.S.A.R. 1999. Effect of silvicultural treatment on growth and mortality of rainforest in Surinam over long periods Forest. Ecology and Management, 124: $123-135$.

He, F.; Legendre P.; Lafrankie, J.V. 1997. Distribution patterns of tree species in a Malaysian tropical rain forest. Journal of Vegetation Science, 8: 105-114.

Holdridge, L.R.; Grenke, W.C.; Hatheway, W.H.; Liang, T.; Tosi, J.A. 1971. Forest Environments in Tropical Life Zones-A Pilot Study. Pergamon Press, New York. 747 pp.

Jackson, S.M.; Fredericksen, T.; Malcolm, J.R. 2002. Area disturbed and residual stand damage following logging in a Bolivian tropical forest. Forest Ecology and Management, 166: 271-283.

Lusk, C.; Ogden, J. 1992. Age structure and dynamics of a podocarpbroadleaf forest in Tongariro National Park, New Zealand. Journal of Ecology, 80: 379-393.

Magnusson, W. E.; de Lima, O.P.; Reis, F.Q.; Higuchi, N; Ramos, J.F. 1999. Logging activity and tree regeneration in an Amazonian forest. Forest Ecology and Management, 113: 67-74.

Mast, J.N.; Veblen, T.T. 1999. Tree spatial patterns and stand development along the pine-grassland ecotone in the Colorado Front Range. Canadian Journal Forest Research, 29: 575-584.

Mostacedo, B; Fredericksen, T. 1999. Regeneration status of important tropical forest tree species in Bolivia: assessment and recommendations. Forest Ecology and Management, 124: $263-273$.

Pariona, W.; Fredericksen, T.S.; Licona, J.C. 2003. Natural regeneration and liberation of timber species in logging gaps in two Bolivian tropical forests. Forest Ecology and Management, 181: 313-322.

Pena-Claros,M; Peters, E.M.; Justiniano, M.J, Bongers, F; Blate, G.M.; Fredericksen, T.S; Putz, F.E. 2008. Regeneration of commercial tree species following silvicultural treatments in a moist tropical forest. Forest Ecology and Management, 225: 1283-1293.

Peres, C.A; Baider, C. 1997. Seed dispersal, spatial distribution and population structure of Brazilnut tres (Bertholletia excelsa) in southeastern Amazonia. Journal of Tropical Ecology, 13: 595-616.

Pimentel, L, A; Pimentel, L. O.; Magnusson, W. E.; Higuchi, N.; Reis, F. Q. 2002. Regeneration of five commercially-valuable tree species after experimental logging in an amazonian forest. Revista Arvore, 26(5): 567-571.

Rivas, C.J.; Aguirre, C.; Jiménez, P.; Corral, R. 2005. Un análisis del efecto del aprovechamiento forestal sobre la diversidad estructural en el bosque mesófilo de montańa "El Cielo», Tamaulipas, México. Sistemas y Recursos Forestales, 14(2): 217-228.

Rose, S.A. 2000. Seeds, seedlings and gaps—size matters. A study in the tropical rain forest of Guyana. In: Tropenbos Guyana Series 9, Tropenbos International, Wageningen. $176 \mathrm{pp}$.

Seidler, T.G.; Plotkin, J.B. 2006. Seed dispersal and spatial pattern in tropical trees. PLoS Biology, 4(11): 2132-2137. 
Schiotz, M.; Boesen, M.V.; Nabe-Nielsen, J.; Sørensen, M.; Kollmann, J. 2006. Regeneration in Terminalia oblonga (Combretaceae)—A common timber tree from a humid tropical forest (La Chonta, Bolivia). Forest Ecology and Management, 225: 306-312.

Stewart, G.H.; Rose, A.B. 1990. The significance of life history strategies in the developmental history of mixed beech (Nothofagus) forests, New Zealand. Vegetatio, 87: 101-114.

Turner, D.P.; Franz, E.H. 1985. Size class structure and tree dispersion patterns in old-growth cedar-hemlock forest of the northern Rocky Mountains (USA). Oecologia, 68: 52-56.

Upton, G.J.G.; Fingleton, B. 1985. Spatial Data Analysis by Example, Vol. 1. Point Pattern and Quantitative Data. Wiley, Chichester. 410 pp.
Van Rheenen, J; Boot, R; Werger, M; Ulloa, M. 2004. Regeneration of timber trees in a logged tropical forest in North Bolivia. Forest Ecology and Management, 200: 39-48.

Ward, J.S.; Parker, G.R.; Ferrandino, F.J. 1996. Long-term spatial dynamics in an old-growth deciduous forest. Forest Ecology and Management, 83: 189-202.

Zonta, A. 1999. Plan General de Manejo, Comunidad 12 de octubre. IPHAE, Riberalta, Bolivia. pp. 50.

Zuidema, P.A., 2003. Demography and management of the nut tree (Bertholletia excelsa) PROMAB, Scientific Series 6. pp. 118.

Recebido em 12/06/2009

Aceito em 13/11/2009 
